\title{
The MRE11/RAD50/NBS1 complex destabilization in Lynch-syndrome patients
}

\author{
Aster Alemayehu ${ }^{1}$ and Ivana Fridrichova*,1 \\ ${ }^{1}$ Laboratory of Cancer Genetics, Cancer Research Institute of Slovak Academy of Sciences, Bratislava, Slovakia
}

Lynch syndrome is an inherited disease leading to the development predominantly of colorectal cancer (CRC). The crucial cause is malfunction of DNA mismatch repair that is characterized by high level of microsatellite instability; however, new knowledge of two MSI modes (types A and B) suggests a more complex molecular basis of this syndrome. To investigate, whether the extensive alterations in individual MSI markers (type B) can indicate the potential deficiency of DNA double-strand break (DSB) repair in Lynch-syndrome-related tumours, we evaluated the MSI type and alterations in the MRE11 and RAD50 repeats that are associated with the reduced protein expression and functional impairment of the MRE11RAD50-NBS1 (MRN) complex. Of 27 CRCs, 21 samples manifested type B in at least one MSI + marker. From type B tumours, the genetic alterations were identified in $16(76 \%)$ samples; seven, one and eight cases manifested mutations in MRE11, RAD50 and both genes, respectively. However, predominantly biallelic MRE 11 alterations with simultaneously developed RAD50 mutations impaired the protein expressions with different intensity and location in tumour. Of six tumours presenting changes $\leqslant 6$ bp (type A), in four samples identical alterations and protein expressions were observed. Moreover, in two patients with different MSI types germline insertions in the MRE11(T) $)_{11}$ were found. Overall, our findings indicate the absence of significant association between type B MSI and MRE11 or RAD50 mutations in tumours of Lynch-syndrome patients, but a subset of them manifested causal mutations for MRN destabilization that could lead to the additional defect in DSB repair.

European Journal of Human Genetics (2007) 15, 922-929; doi:10.1038/sj.ejhg.5201858; published online 30 May 2007

Keywords: Lynch syndrome; microsatellite instability; two types of MSI; MRE11; RAD50

\section{Introduction}

Lynch syndrome (hereditary non-polyposis colorectal cancer - HNPCC) is an autosomal-dominant disease that results in several types of cancer, predominantly those located in the colon, rectum or endometrium. It is generally accepted that tumour development is initiated by DNA mismatch repair (MMR) malfunction that is caused by germline mutations in MMR genes, mostly in MLH1 and MSH2. The significant hallmark of MMR defects

*Correspondence: Dr I Fridrichova, Laboratory of Cancer Genetics, Cancer Research Institute of Slovak Academy of Sciences, Vlarska 7, 83391 Bratislava, Slovakia. Tel: +421 259327 208; Fax: +421 259327 250; E-mail: Ivana.Fridrichova@savba.sk

Received 21 September 2006; revised 4 April 2007; accepted 24 April 2007; published online 30 May 2007 is the high level of instability in repetitive sequences, microsatellites (MSI-H).

The introduction of a new fluorescent technique enables the recognition of the two types of instability in dinucleotide microsatellites of different origin. MSI marker of type A was characterized by short insertions and deletions of up to $6 \mathrm{bp}$, as a direct consequence of MMR deficiency. Type B is represented by more extensive changes of $\geqslant 8 \mathrm{bp}$ that are caused by an additional molecular mechanism other than MMR inactivation, probably by inappropriate recombinational DNA repair. The former type was frequently observed in mice, human cell lines and patients suffered from rectal tumours with low levels of MSI (MSI-L), ${ }^{1,2}$ the latter in various malignancies, including Lynch-syndrome cancers. $^{3}$ 
Maintenance of genomic integrity during the DNA replication process is provided by a group of BRCA1associated proteins of the BASC supercomplex that is considered to be a sensor for DNA damage. ${ }^{4}$ The components of this complex are MMR proteins and also a heterotrimer MRE11-RAD50-NBS1 (MRN) which is essential for DNA double-strand break (DSB) repair performing by homologous recombination (HR) or non-homologous end joining (NHEJ). ${ }^{5}$ Recent evidence indicates that multiple activities of several proteins occur in different pathways, such as the role of MSH2 protein in signalling and cell-cycle regulation of ATM and MRN complex ${ }^{6}$ or direct association of MRE11 and MLH1 proteins, which can result in mutual cooperation during DNA-damage detection, signalling and repair. ${ }^{7}$

Relation between the malfunction of MMR and aberrant recombinational DNA repair in animal and human cancer cells has previously been observed. Mouse MSH2-deficient cells have lost the dependence of complete identity between interacting DNA sequences in the process of HR repair. ${ }^{8}$ Similarly, in mouse embryonic stem cells and human tumour cell lines that have the MSH2 mutation, the barrier to recombination between diverged substrates was relaxed and DSBs were repaired predominantly by nonreciprocal HR - gene conversion. , $^{9} 10$

Mismatch-repair-deficient tumours are characterized by widespread changes in the number of microsatellites accumulating in both coding and non-coding sequences of many human genes. Genetic alterations occurring in coding repeats result in frameshift mutations and the expression of inactive proteins. The changes in intronic microsatellites can also lead to functional changes, if they are located in regulatory regions of genes. In a series of unstable gastric and colorectal cancers (CRCs), mono- or biallelic deletions in the poly $(\mathrm{T})_{11}$ within MRE11 intron 4 were detected that cause aberrant splicing signals, skipping of exon 5, introduction of a premature stop codon and generation of a truncated protein. However, only larger and biallelic shortening corresponds with the strong reduction or absence of MRE11 protein expression. ${ }^{11,12}$ Additionally, the results of two independent studies have documented the high frequency of frameshift mutations in the $(\mathrm{A})_{9}$ repeat that is located in $R A D 50$ exon 13 , but no mutations were found in the $(\mathrm{A})_{7}$ tract of the NBS1 gene in primary gastrointestinal carcinomas with the MSI-H phenotype. ${ }^{13,14}$ Furthermore, in several MMR-deficient cell lines with MRE11 biallelic alterations and monoallelic changes in $R A D 50$ repeat, the significant reduction of both proteins and corresponding mRNAs were identified. In addition, these cell lines showed an impaired NHEJ. ${ }^{15}$ Moreover, the results of in vitro studies predict that the MRE11 gene could be a functional component of MMR pathway, as the presence of MRE11 mutations increased MSI in both mono- and dinucleotide repeats. Disruption of MLH1-MRE11 interactions might represent an alternative molecular basis of MLH1 mutations in a subset of Lynchsyndrome patients. ${ }^{16}$

Previously characterized MSI in cancers of Lynchsyndrome patients manifested mainly type B MSI, as type A MSI was not recognized by formerly utilized isotopiclabelled PCR assay. ${ }^{17}$ However, more extensive alterations of dinucleotide repeats described in MSI + cancer cell lines and unselected gastrointestinal tumours could reflect the deficiency of recombinational DNA repair. To investigate, whether or not type B MSI can indicate the potential deficiency of DSB repair in tumours related to Lynch syndrome, we evaluated the MSI type and alterations in repetitive sequences of the MRE11 and RAD50 genes with corresponding protein expressions in hereditary CRCs.

\section{Patients and methods}

In total, the samples from 33 patients were collected for our study; of these, 27 MSI-H tumours developed in 25 Lynch-syndrome patients and single CRCs in eight patients with MSI-L phenotype in an average age of $38.3 \pm 8.7$ and $38.4 \pm 11$ years, respectively. All persons fulfilled Amsterdam criteria or Bethesda Guidelines. In most of the patients with MSI-H phenotype, the MMR germline mutations were detected by genomic sequencing. ${ }^{18,19}$ The identification of inherited genetic alterations in the rest of patients by sequencing or MLPA analysis for recognition of long deletions is still in process.

The evaluation of MSI type was performed on matched DNA samples (normal/tumour). The samples were analysed by a high-resolution fluorescent-labelling method using 5-9 highly polymorphic dinucleotide markers: D2S378, D2S123, D2S391, D3S1561, D3S1611, D3S3685, D5S346, D17S250 and D18S34, as described previously. ${ }^{20}$ Forward primer for each MSI marker was labelled on the $5^{\prime}$-end by fluorescent dye (FAM, HEX or NED). The PCR products were analysed using ABI PRISM 310 Genetic Analyzer (Applied Biosystems, Foster City, CA, USA) and collected data were evaluated by GeneScan software. Tumours were classified as MSI-H if at least 30\% of markers manifested an instability, and as MSI-L if less than 30\% positive MSI markers were identified. The MSI + markers were characterized by length of alteration: type A manifested changes of $\leqslant 6 \mathrm{bp}$ and type $\mathrm{B}$ with insertions or deletions of $\geqslant 8 \mathrm{bp}$. Tumours were classified as type B if at least one $\mathrm{MSI}+$ marker manifested type B MSI.

The impairment of the MRE11 and RAD50 gene expressions was evaluated by genetic analyses of mononucleotide intronic repeats $(\mathrm{T})_{11}$ within the polypyrimidine stretch/ accessory splicing signal and exonic $(\mathrm{A})_{9}$ tract, respectively, together with immunohistochemical (IHC) analyses of relevant protein expressions. Insertions or deletions were examined by two methods: fragment analysis and genomic sequencing. For fragment analysis, the multiplex PCRs 
were carried out in a $30 \mu \mathrm{l}$ reaction mixture that contained $100 \mathrm{ng}$ purified DNA, $1 \times$ QIAGEN Multiplex PCR Master Mix and $0.2 \mu \mathrm{M}$ of each primer for the MRE11 and RAD50 genes. The PCR program was performed as described in the MSI cycling protocol, with annealing temperature of $59^{\circ} \mathrm{C}^{21}$ PCRs for genomic sequencing were carried out in a $30 \mu \mathrm{l}$ reaction mixture, containing $100 \mathrm{ng}$ purified DNA, $0.33 \mu \mathrm{M}$ of each forward and reverse primer, $200 \mu \mathrm{M} \mathrm{dNTP}$, $1.5 \mathrm{mM} \mathrm{MgCl}$, $1 \times$ DynaZyme EXT buffer and $1 \mathrm{U}$ DynaZyme EXT DNA Polymerase (Finnzymes, Espoo, Finland). The PCR program consisted of an initial denaturation at $94^{\circ} \mathrm{C}$ for $2 \mathrm{~min}$, followed by 35 cycles of $94^{\circ} \mathrm{C}$ for $30 \mathrm{~s}, 59^{\circ} \mathrm{C}$ for $30 \mathrm{~s}$ and $72^{\circ} \mathrm{C}$ for $1 \mathrm{~min}$. The modification of five cycles of $94^{\circ} \mathrm{C}$ for $1 \mathrm{~min}, 59^{\circ} \mathrm{C}$ for $2 \mathrm{~min}$ and $72^{\circ} \mathrm{C}$ for $3 \mathrm{~min}$ with a following 25 cycles of $94^{\circ} \mathrm{C}$ for $30 \mathrm{~s}, 59^{\circ} \mathrm{C}$ for $2 \mathrm{~min}$ and $72^{\circ} \mathrm{C}$ for $90 \mathrm{~s}$ was used for paraffin blocks. The final extension was performed at $72^{\circ} \mathrm{C}$ for $10 \mathrm{~min}$. The primers used in both methods are summarized in Table $1 .^{22}$ The sequencing reactions were performed according to the standard protocols.

Expressions of MRE11 and RAD50 proteins were analysed by IHC assay. Briefly, formalin-fixed, paraffin-embedded tissues ( $4 \mu \mathrm{m}$ sections) were mounted on silanized slides, deparaffinized and rehydrated. Antigen retrieval was accomplished by heating the sections in a pressure cooker at $120^{\circ} \mathrm{C}$ for $2 \mathrm{~min}$ in $10 \mathrm{~mm}$ citrate-buffered solution $(\mathrm{pH}$ 6.0). Dako peroxidase blocking reagent and goat serum were sequentially used to suppress nonspecific staining due to endogenous peroxidase activity and unspecific binding of antibodies, respectively. The incubation of sections was performed at $4{ }^{\circ} \mathrm{C}$ for $24 \mathrm{~h}$ with $1: 3000$ and $1: 500$ dilutions of the MRE11 and RAD50 antibodies (Novus Biological, Littleton, CO, USA), respectively. After washing, anti-rabbit secondary antibodies conjugated to peroxidase-labelled polymer (DAKO EnVision + kit, DakoCytomation, Glostrup, Denmark) were applied for $30 \mathrm{~min}$ at RT, and the peroxidase activity was developed by incubation with $3,3^{\prime}$ diaminobenzidine chromogen solution (Dako). The sections were then counterstained with haematoxylin. The protein expression in tumour tissue was classified as negative if the nuclear staining was weak or none in contrast with normal stained colonocytes and stroma cells in the samples of the same patient.

\section{Results \\ Two types of MSI in Lynch-syndrome patients}

All 27 MSI-H colorectal carcinomas showed MSI in more than $38 \%$ of evaluated markers. Of 21 tumour samples manifesting MSI + markers of both $\mathrm{A}$ and $\mathrm{B}$ types simultaneously, type B in more than $50 \%$ of repeats was found in seven cases only. The incidence of type $B$ varied from 14.3 to $100 \%$. Regardless of the frequency, all 21 tumours were classified as type B. The tumour samples from six individuals manifested exclusively type A markers and were assigned as type A MSI. Two of the patients developed synchronous and metachronous CRCs with a similar high frequency of type B MSI (57 and 62.5\%), and with different MSI type (exclusively markers of type B or A), respectively. The frequencies of MSI types in tumours with an MSI-H phenotype are summarized in Table 2. Cancers of $\mathrm{A}$ and $\mathrm{B}$ type were predominantly located in the left-sided (5/6) and right-sided (15/21) colon, respectively. Similarly, the left location of CRCs was found in five out of seven patients with MSI-L phenotype who manifested subtle microsatellite alterations (type A) in one or two MSI+ markers. One person in this group exhibited a single marker with type B MSI in a colorectal tumour at an unknown location.

\section{Mutations in the MRE11 and RAD50 genes and protein expressions}

Two crucial genes for the cellular response to DNA damage and the maintenance of chromosomal integrity, MRE11 and $R A D 50$, were chosen for investigation of potential malfunction of DSB repair in CRCs of Lynch-syndrome patients. Both genes contain the splicing-site region or exonic mononucleotide repeats, the changes of which lead to impaired protein function. The numeric alterations of the MRE11 ( T $)_{11}$ and RAD50 (A) $)_{9}$ tracts were analysed by two methods: fragment analysis and genomic sequencing. In $96 \%$ of all examined tumour samples, the identical results obtained by these assays were found. However, the presence of changes in microsatellites was more demonstrative by using the fragment analysis than genomic sequencing in many cases.

From 21 CRCs with type B MSI, the genetic alterations were identified in 16 (76\%) samples; seven, one and eight

Table 1 Oligonucleotide sequences of the primers

\begin{tabular}{ll}
\hline Primer name & Oligonucleotide sequence $\left(5^{\prime}-3^{\prime}\right)$ \\
\hline FA-MRE11 - F & FAM-TGGAGGAGAATCTTAGGGAAAA \\
FA-MRE11 - R & AATTGAAATGTTGAGGTTGCC \\
FA-RAD50 - F & HEX-TGCGACTTGCTCCAGATAAA \\
FA-RAD50 - R & GCTACATGTACAGTGAAGGTAAATCC \\
GS-MRE11 - F & TTGAAATGAATTGTCGCCTATG \\
GS-MRE11 - R & AATTGAAATGTTGAGGTTGCC \\
GS-RAD50 - F & CCCCGTTGTCAGAGAGTTT \\
GS-RAD50 - R & GGCATGAGATGGGTACCTTTT \\
\hline
\end{tabular}

FA, fragment analysis; GS, genomic sequencing. 
Table 2 Frequency of types A and B in MSI-H tumours

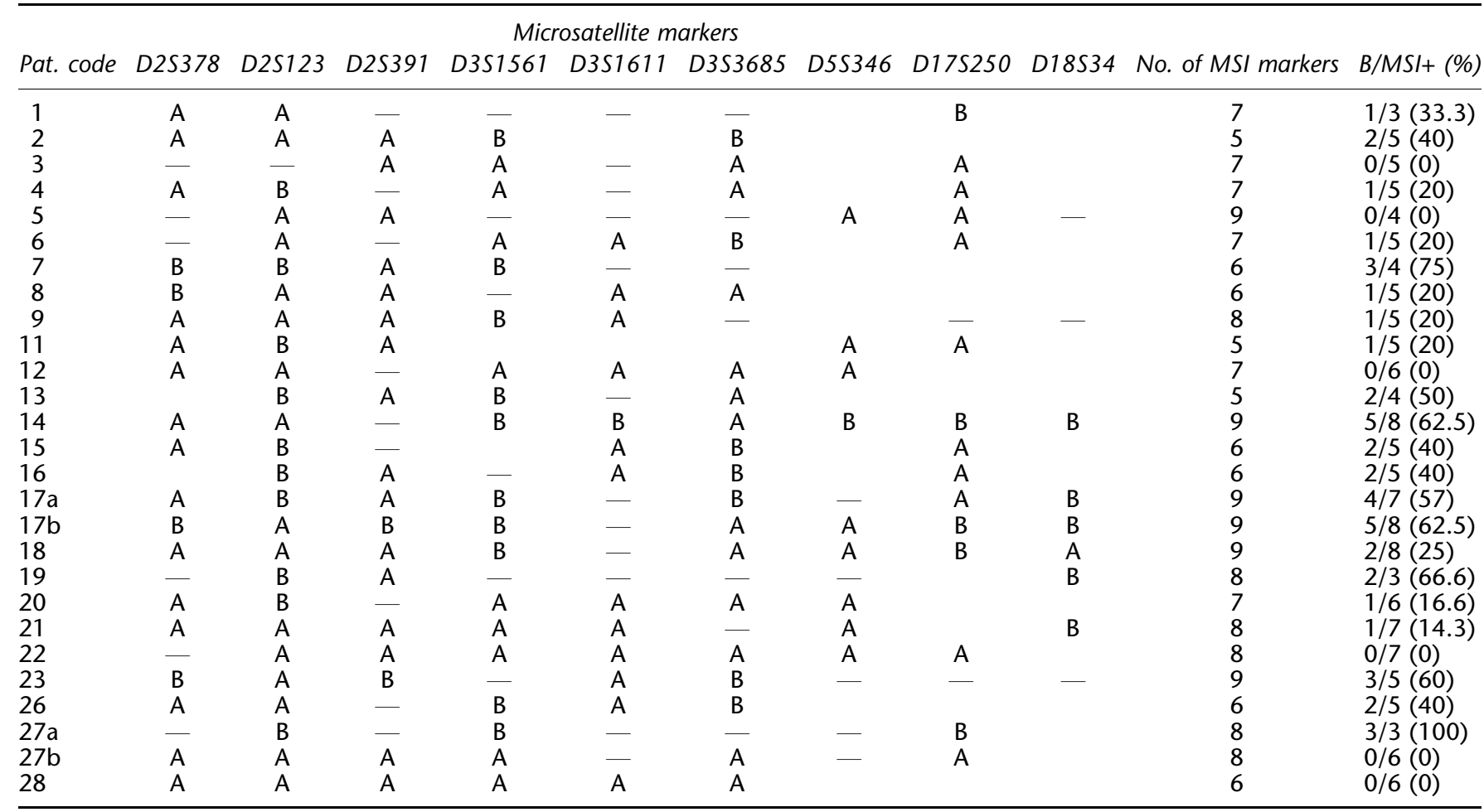

$17 \mathrm{a}, \mathrm{b}$ and $27 \mathrm{a}, \mathrm{b}$ - codes of patients with synchronous and metachronous colorectal cancers, respectively.

cases manifested mutations in MRE11, RAD50 and both genes, respectively. Out of six tumours with type A MSI, in four samples mutations in both evaluated genes were observed. No changes in both repeats were seen in five and two carcinomas with type B and A MSI, respectively.

In the MRE11 (T) 11 repeats of type B tumours, the shortening and expansions from 1-3 units were observed. Biallelic mutations and monoallelic deletions were found in eight and seven tumour samples, respectively. Expression of MRE11 protein was immunohistochemically analysed in 14 MSI-H tumours. The reduction or absence of MRE11 protein expression was identified with variable distribution from several tumour glands to the whole sample in the most of evaluated tumour tissues with both affected alleles. Examples of IHC results in two cancers with biallelic MRE11 mutations are shown in Figure 1. However, the monoallelic MRE11 changes influenced the stability of the protein only in one case.

Monoallelic mutation in $R A D 50(A)_{9}$ sequence was observed in eight samples with B type MSI, on the other hand biallelic alteration was found only in one case. The RAD50 protein expressions were decreased simultaneously with MRE11 reduction in six cases regardless of a number of affected alleles of RAD50 gene. Similar relation between alterations in both microsatellites and decreasing of protein expressions were evidenced in cancers with type
A MSI. The results of microsatellite alterations and protein expressions are summarized in Table 3.

In patient 20 with type B MSI cancer one allele with $1 \mathrm{bp}$ deletion, together with $1 \mathrm{bp}$ insertion in the other allele simultaneously in MRE11 and RAD50 repeats were identified. However, the identical biallelic alteration, but only in the MRE11 gene, was observed in individual (code 28) suffered from CRC with type A MSI. Monoallelic insertions in the MRE11 gene, described above, are confirmed to be inherited, because the same findings were seen in their blood samples. Electrophoretograms of fragment analysis and genomic sequencing of patients affected by microsatellite changes in both genes (codes 2 and 20) are shown in Figure 2; later patient is also a carrier of the MRE11 germline mutation.

\section{Type MSI and MRN complex destabilization}

The DSB repair efficiency depends on the formation of the functional MRN protein complex. The impairment of HR or NHEJ pathways, in which the MRN protein complex plays a crucial role, can be caused by mutations in any of the three components of this complex. In MMR-defective cells, the numeric alterations of repetitive sequences as a consequence of DNA-repair malfunction have been predominantly accumulated. Deletions and very probably also insertions in the MRE11 (T) $)_{11}$ and $R A D 50(\mathrm{~A})_{9}$, as seen in 


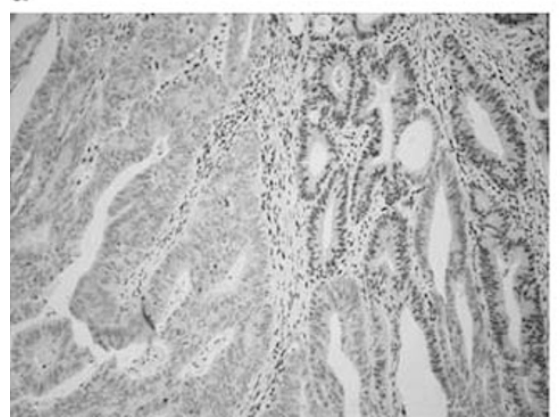

b

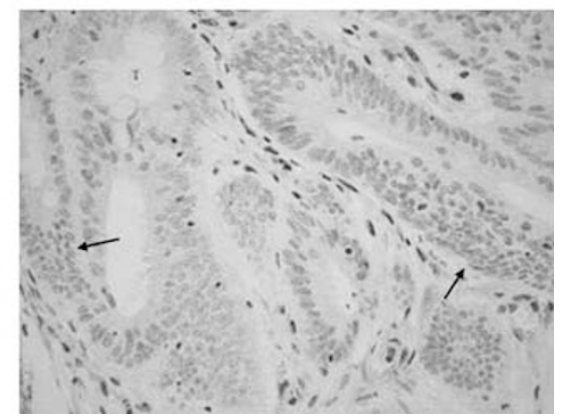

Figure 1 IHC staining with anti-MRE11 antibody. (a) Tumour tissue displaying biallelic MRE11 (T) $)_{11}$ deletion (code 11) showed a loss of nuclear expression of MRE11 approximately in 50\% of analysed section. The tumour areas with negative and positive MRE11 staining are evident in presented slot. (b) The very weak nuclear staining of MRE11 protein only in several tumour glands (indicated by arrows) was found in sample 20 manifesting one allele with $1 \mathrm{bp}$ shortening, together with $1 \mathrm{bp}$ expansion of the other allele in $(\mathrm{T})_{11}$ repeat (magnification $\times 100$ on both pictures).

Table 3 Microsatellite changes in MRE11 and RAD50 genes and expression of relevant proteins in MSI-H tumours

\begin{tabular}{|c|c|c|c|c|c|c|c|c|c|}
\hline \multirow[t]{2}{*}{ Pat. code } & \multirow[t]{2}{*}{ MSI type } & \multicolumn{2}{|c|}{ Fragment analysis } & \multicolumn{2}{|c|}{ Genomic sequencing } & \multicolumn{2}{|c|}{ Microsatellite changes ${ }^{\mathrm{a}}$} & \multicolumn{2}{|c|}{ Protein expression ${ }^{\mathrm{b}}$} \\
\hline & & MRE11 & RAD50 & MRE11 & RAD50 & $\operatorname{MRE} 11(\mathrm{~T})_{11}$ & $R A D 50(\mathrm{~A})_{9}$ & MRE11 & RAD50 \\
\hline 1 & B & - & - & - & - & wt & wt & & \\
\hline 2 & B & + & + & + & + & $1 \mathrm{del} / 2 \mathrm{del}$ & $1 \mathrm{del}$ & $\mathrm{NI}$ & + \\
\hline 3 & $A$ & - & - & - & - & wt & wt & & \\
\hline 4 & B & + & + & + & + & $1 \mathrm{del}$ & $1 \mathrm{del}$ & $-^{c}$ & ${ }^{d}$ \\
\hline 5 & $A$ & + & + & + & + & $1 \mathrm{del}$ & 1 ins & $+^{\mathrm{e}}$ & + \\
\hline 6 & B & - & - & - & - & wt & wt & & $T$ \\
\hline 7 & B & + & + & - & + & $1 \mathrm{del} / 2 \mathrm{del}$ & 1 ins & $\pm^{f}$ & $\pm^{g}$ \\
\hline 8 & $\mathrm{~B}$ & - & + & ND & + & wt & $1 \mathrm{del}$ & & \\
\hline 9 & B & - & - & - & - & wt & wt & + & + \\
\hline 11 & B & + & - & + & - & $1 \mathrm{del} / 3 \mathrm{del}$ & wt & $\pm^{f}$ & $\pm^{g}$ \\
\hline 12 & A & - & - & - & - & wt & wt & + & + \\
\hline 13 & B & + & + & + & + & $1 \mathrm{del}$ & $1 \mathrm{del}$ & $\mathrm{NI}$ & + \\
\hline 14 & $\mathrm{~B}$ & + & ND & ND & + & $1 \mathrm{del} / 2 \mathrm{del}$ & $1 \mathrm{del}$ & + & + \\
\hline 15 & B & + & - & + & - & 1del & wt & + & \\
\hline 16 & B & - & - & ND & - & wt & wt & & \\
\hline $17 a$ & B & + & + & + & + & $1 \mathrm{del}$ & $1 \mathrm{del}$ & & \\
\hline $17 b$ & B & + & - & + & - & $1 \mathrm{del}$ & wt & & \\
\hline 18 & B & + & - & + & - & $1 \mathrm{del}$ & wt & + & \\
\hline 19 & B & + & - & + & - & $1 \mathrm{del} / 2 \mathrm{del}$ & wt & & \\
\hline 20 & B & + & + & + & + & $1 \mathrm{del} / 1$ ins $^{\mathrm{h}}$ & $1 \mathrm{del} / 1 \mathrm{ins}$ & $t^{c}$ & $+^{c, g}$ \\
\hline 21 & B & + & - & + & - & $1 \mathrm{del}$ & wt & & \\
\hline 22 & A & - & + & - & + & wt & 1 ins & & \\
\hline 23 & B & - & - & - & - & wt & wt & & \\
\hline 26 & B & + & - & + & - & $1 \mathrm{del} / 2 \mathrm{del}$ & wt & & \\
\hline $27 a$ & B & + & - & + & - & $1 \mathrm{del} / 2 \mathrm{del}$ & 1 del & $-^{\mathrm{c}}$ & ${ }^{d}$ \\
\hline $27 b$ & $A$ & + & - & + & - & $1 \mathrm{del} / 2 \mathrm{del}$ & wt & $-^{c}$ & ${ }^{d}$ \\
\hline 28 & $A$ & + & - & + & - & $1 \mathrm{del} / 1$ ins $^{\mathrm{h}}$ & wt & & \\
\hline
\end{tabular}

+ or -, presence or absence of changes in MRE11 $(\mathrm{T})_{11}$ or $R A D 50(\mathrm{~A})_{9}$ repeats in tumour samples; ND, not detected results for DNA sample degradation in fragment analysis and genomic sequencing.

${ }^{a}$ Numeric changes in microsatellites in tumour samples evaluated by fragment analysis; wt, wild-type; 1 del, 1 bp deletion; 2 del, 2 bp deletion; 1 ins, $1 \mathrm{bp}$ insertion; del/del or del/ins, biallelic deletion or combination of deletion and insertion.

b Protein expression in tumour samples analysed by immunohistochemistry; NI, not informative for weak staining in the normal and tumour epithelial cells.

${ }^{\mathrm{C}}$ Very weak staining in some tumour glands, but always less than in normal cells.

${ }^{\mathrm{d}}$ Cytoplasmic staining maintained.

e One region is clearly negative.

fSome regions are positive, some are negative.

${ }^{9}$ The nuclear expression is markedly lost in the same regions where MRE11 is not expressed.

${ }^{\mathrm{h}}$ Monoallelic insertion of $1 \mathrm{bp}$ was presented also in blood sample. 
a
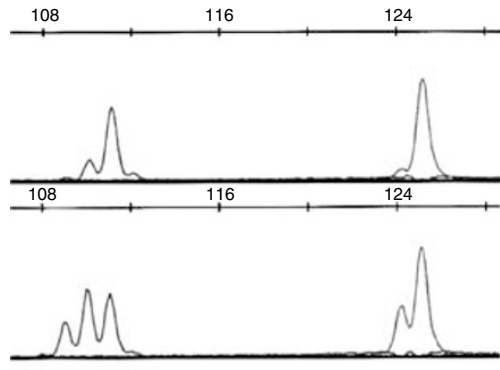

108

116
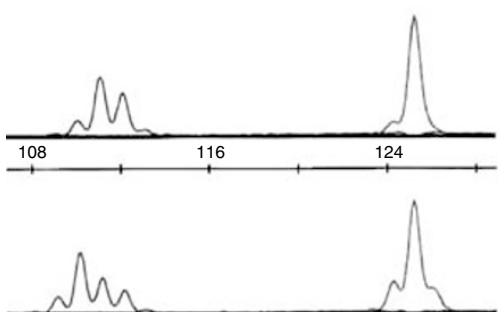

b

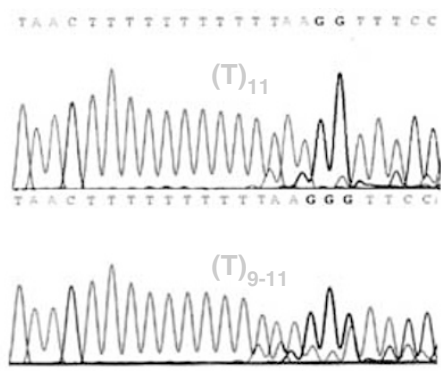

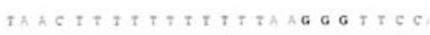
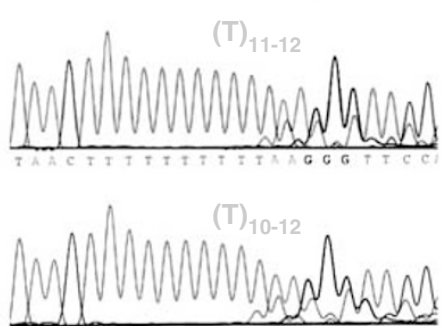

C
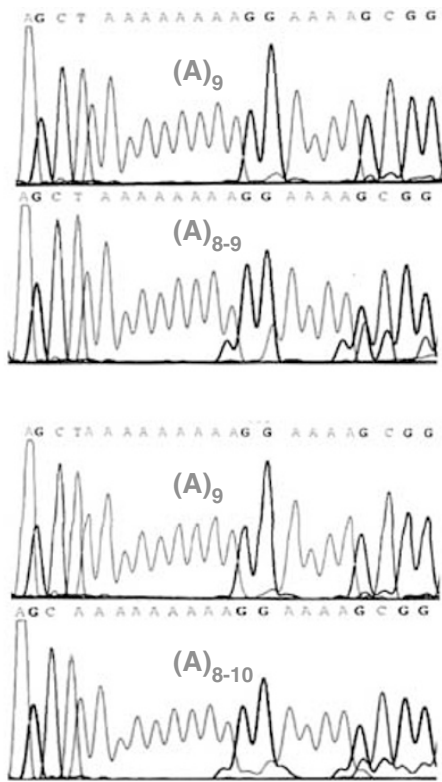

Figure 2 Numeric changes in the MRE11 ( $\mathrm{T})_{11}$ and $\operatorname{RAD50}(\mathrm{A})_{9}$ microsatellites. (a) Fragment analysis of the MRE11 (left) and RAD50 (right); genomic sequencing of MRE11 (b) and RAD50 (c). In the upper two rows, the results of normal and tumour samples of case 2 are presented. Biallelic change in MRE11 (deletion of 1 and $2 \mathrm{bp}$ ) and monoallelic change in RAD50 (deletion of $1 \mathrm{bp}$ ) are clearly visible from fragment analysis (a). The shortening of 1 and $2 \mathrm{bp}$ in $(T)_{11}$ repeat $(\mathbf{b})$ and $1 \mathrm{bp}$ in $(A)_{9}$ repeat (c) is detectable by sequencing that corresponds to the results of fragment analysis. In the bottom two rows, the results of normal and tumour samples of case 20 are presented. Insertion of 1 bp in MRE11 is detectable in both normal and tumour samples, $1 \mathrm{bp}$ shortening only in later one $(\mathbf{a}, \mathbf{b})$. Deletion in first allele and insertion in second one are presented in RAD50 gene of the tumour sample (a); that was recognized very weakly by sequencing (c); therefore, the changes were developed only in small part of analysed cells.

tumour samples of Lynch-syndrome patients in present study, can result in aberrant splicing signals and premature stop codons of the MRE11 and RAD50 genes, respectively. However, the incidence of these microsatellite changes which lead to the reduction of MRE11 or RAD50 protein expressions is not specific for particular type of MSI. We concluded that our results did not confirm the relevant connection between type B MSI and destabilization of MRN complex in Lynch-syndrome cancers, but a subset of patients unambiguously presented causal mutations for possible additional defect in DSB repair.

\section{Discussion}

It is generally accepted that the malfunction of DNA MMR induced by germline mutations is the causal defect in patients suffering from HNPCC, which was recently renamed according to the historical name of the disease as Lynch syndrome. ${ }^{23}$ New knowledge in the two different modes of microsatellite changes indicates that the molecular basis of this syndrome seems to be more complex. The subtle microsatellite alterations in numerous repeats (type A) were considered to be a direct consequence of defective MMR as was found in previous studies in mouse and human cell lines with identified MMR mutations. ${ }^{1,24}$
In tumour samples with more extensive changes in MSI markers (type B) apart from MMR deficiency, DNA recombinational repair could also be inhibited. ${ }^{3}$

Here, we showed four Lynch-syndrome patients with exclusively type A MSI who manifested also the changes in MRE11 and RAD50 genes predisposing to additional repair defect. On the other hand, in most patients suffered from CRCs with MSI-L phenotype, type A MSI was found in present study as well as in other laboratories. ${ }^{2}$ However, the origin of type A in these cancers seems to be independent of MMR defects, because it has been accepted that MSI-L CRCs are developed by a distinct carcinogenetic pathway. ${ }^{25}$ Additionally, the microsatellite repeat slippage was also observed in normal cells with a frequency of about every 1000-10000 cell divisions and the clonal nature of cancers would make this slippage detectable during tumorigenesis that probably occurred in CRCs with the MSI-L phenotype. ${ }^{26}$

The present study documented the relationship between MSI type and tumour location in Lynch-syndrome patients. Carcinomas with A and B type were found predominantly in the left and right side of the colon, respectively, as was published previously. ${ }^{2}$ However, this phenotypical characterization was the only difference that we observed between cancers with A or B type changes in microsatellites. 
Our results showed that in unstable markers predominantly type B MSI was observed in 78\% Lynch-syndromerelated cancers, while the presence of alterations in MRE11 and $R A D 50$ repeats was not specific for those samples. This conclusion was significantly confirmed by findings in two patients with synchronous or metachronous cancers, in which the different and identical changes in the MRE11 $(\mathrm{T})_{11}$ and $R A D 50(\mathrm{~A})_{9}$ repeats were found in two carcinomas with the same and different MSI type, respectively (codes 17 and 27).

The frequency of biallelic deletions in the MRE11 $(\mathrm{T})_{11}$ repeats varied from 25.9 to $38.8 \%$ in unselected gastric or CRCs with the MSI-H phenotype, but in familial colorectal cases, $55.6 \%$ was shown in their cancers. ${ }^{11,12}$ From our set of tumours that were related to Lynch syndrome, regardless of MSI type, $37 \%$ of cases presented the biallelic changes, including insertions. In contrast to other investigators, we were first, who described the germline insertions in the repeated unit of MRE11 gene.

In other studies, the frameshift mutations in the $(\mathrm{A})_{9}$ repeat of $R A D 50$ gene in $31-46 \%$ of microsatellite-unstable gastric and colon carcinomas were found ${ }^{13,14}$ that corresponded to our results including mono- and biallelic alterations (40.7\%).

The IHC analyses showed the variable distribution of cells with reduced MRE11 protein expression. Very weak MRE11 staining in the most of samples with biallelic mutations was located in some tumour glands or in one region or in approximately half of evaluated section of tumour sample. It is widely known that the development of MSI phenotype is a process originating with MMR inhibition. Therefore, the gradual accumulation of MSI alterations in several parts of heterogeneous tumour tissue can be the reason of variable findings of protein expressions. The RAD50 expression was simultaneously reduced in cells or regions with aberrant MRE11 expression, regardless of the character of microsatellite changes. Similar reciprocal influence of MRE11 and RAD50 proteins stability was observed in CRC cell lines and urothelial cell carcinomas with MSI phenotype. ${ }^{15,27}$ Recent studies confirmed the necessity of all MRN components in response to DSBs with findings that they stimulates ATM after its binding to broken DNA strands, ${ }^{28}$ while the stability of complex is provided with MRE11 protein. $^{29}$ Taking into account the determining role of MRE11, the lack of RAD50 protein expression in case $27 \mathrm{~b}$ without any MSI changes can be a consequence of MRE11 decreasing. Overall, the microsatellite changes in MRE11 and RAD50 genes with following reduction of protein expression result in MRN destabilization and impairment of DSB repair. However, cooperation between MMR and DSB repair is realized by multiple functions of MSH2, MLH1 and MRE11 genes. ${ }^{8-10,16}$ Moreover, in MLH1 gene an interacting domain (region 495-756 codons) with MRE11 gene was identified, ${ }^{16}$ and in present study two patients (codes 2 and 11) manifested germline deletions in this region of MLH1 gene. ${ }^{18,19}$ It is possible that weak staining of MRE11 protein in patient 2, in both normal and tumour tissues, was affected by the absence of MLH1-MRE11 interaction.

For MSI evaluation in the MRE11 and RAD50 genes, we used two different assays. In heterogeneous tumour tissues, the changes of microsatellites can be developed only in a part of the cancer cells. Fragment analyses allowed the recognition of DNA fragments with a resolution of $1 \mathrm{bp}$ difference in length in a minority of the analysed cell population. On the other hand, sequencing results showed a weak signal from some alleles with various numbers of mononucleotide repeats that were often unrecognized from the background. This observation can be included in the evaluation of patient 7 , in which the opposite results from sequencing in comparison with fragment analysis were seen. Therefore, we consider fragment analysis to be more suitable for MSI evaluations in contrast to several investigators, who have studied the same repeats of the MRE11 or RAD50 genes by sequencing.

In conclusion, our results showed the absence of significant relation between the presence of more extensive alterations of microsatellites, type B MSI, and MRE11 (T) 11 or $R A D 50(\mathrm{~A})_{9}$ mutations in tumour tissues of Lynchsyndrome patients, as the identical biallelic or monoallelic alterations were found equally in type A and B MSI cancers. However, our data indicate that apart from mutation in MMR genes also alterations in key players of DSB repair, MRE11 and RAD50 genes, can be involved in tumorigenesis of Lynch-syndrome-related cancers. Additionally, a subset of patients manifested germline mutations for MRN complex destabilization that might increase a risk for additional DSB repair deficiency and acceleration of genome instability. Moreover, the new knowledge of molecular basis of Lynch syndrome might influence the strategy of therapy.

\section{Acknowledgements}

We thank Dr Giancarlo Marra for helpful discussions and IHC evaluation, Ritva Haider for performing IHC analyses and Dr Boris Rychly for preparing tumour sample pictures. The study was supported by the following grants: National Program 'Use of Cancer Genomics to Improve the Human Population Health' (contract no. 2003 SP 5102 08 00/028 08 01), Grants no. 2/3088/5, 2/7061/7 from Slovak Grant Agency VEGA and the League Against Cancer Slovakia.

\section{References}

1 Oki E, Oda S, Maehara Y, Sugimachi K: Mutated gene-specific phenotypes of dinucleotide repeat instability in human colorectal carcinoma cell lines deficient in DNA mismatch repair. Oncogene 1999; 18: 2143-2147.

2 Ikeda Y, Oda S, Abe T, Ohno S, Maehara Y, Sugimachi K: Features of microsatellite instability in colorectal cancer: comparison between colon and rectum. Oncology 2001; 61: 168-174.

3 Oda S, Maehara Y, Ikeda Y et al: Two modes of microsatellite instability in human cancer: differential connection of defective 
DNA mismatch repair to dinucleotide repeat instability. Nucleic Acids Res 2005; 33: 1628-1636.

4 Wang Y, Cortez D, Yazdi P, Neff N, Elldge SJ, Qin J: BASC: a super complex of BRCA1 associated proteins involved in the recognition and repair of aberrant DNA structures. Genes Dev 2000; 14: 927-939.

5 van den Bosch M, Bree RT, Lowndes NF: The MRN complex: coordinating and mediating the response to broken chromosomes. EMBO Rep 2003; 4: 844-849.

6 Franchitto A, Pichierri P, Piergentilli R, Crescenzi M, Bignami M, Pallitti F: The mammalian mismatch repair protein MSH2 is required for correct MRE11 and RAD51 relocalization and for efficient cell cycle arrest induced by ionising radiation in G2 phase. Oncogene 2003; 22: 2110-2120.

7 Her C, Vo AT, Wu X: Evidence for a direct association of hMRE11 with the human mismatch repair protein hMLH1. DNA Repair 2002; 1: 719-729.

8 de Wind E, Dekker $M$, Berns $A$, Radman $M$, te Riele $H$ : Inactivation of the mouse Msh2 gene results in mismatch repair deficiency, methylation tolerance, hyperrecombination and preposition to cancer. Cell 1995; 82: 321-330.

9 Elliott B, Jasin M: Repair of double-strand breaks by homologous recombination in mismatch repair-defective mammalian cells. Mol Cell Biol 2001; 21: 2671-2682.

10 Villemure JF, Abaji C, Cousineau I, Belmaaza A: MSH2-deficient human cells exhibit a defect in the accurate termination of homology-directed repair of DNA double-strand breaks. Cancer Res 2003; 63: 3334-3339.

11 Ottini L, Falchetti M, Saieva C et al: MRE11 expression is impaired in gastric cancer with microsatellite instability. Carcinogenesis 2004; 25: 2337-2343.

12 Giannini G, Rinaldi C, Ristori E et al: Mutations of an intronic repeats induce impaired MRE11 expression in primary human cancer with microsatellite instability. Oncogene 2004; 8: 2640-2647.

13 Kim NG, Choi YR, Back MJ et al: Frameshift mutation at coding mononucleotide repeats of the $h R A D 50$ gene in gastrointestinal carcinomas with microsatellite instability. Cancer Res 2001; 61: $36-38$

14 Ikenoue $\mathrm{T}$, Togo $\mathrm{G}$, Nagai $\mathrm{K}$ et al: Frameshift mutation at mononucleotide repeats in RAD50 recombinational DNA repair gene in colorectal cancers with microsatellite instability. Jpn J Cancer Res 2001; 92: 587-591.

15 Koh KH, Kang HJ, Long SL et al: Impaired nonhomologous endjoining in mismatch-deficient colon carcinomas. Lab Invest 2005; 85: $1130-1138$
$16 \mathrm{Vo}$ AT, Zhu F, Wu X et al: hMRE11 deficiency leads to microsatellite instability and defective DNA mismatch repair. EMBO Rep 2005; 6: 438-444.

17 Liu B, Parsons R, Papadopoulos $\mathrm{N}$ et al: Analysis of mismatch repair genes in hereditary non-polyposis colorectal cancer patients. Nat Med 1996; 2: 169-174.

18 Bartosova Z, Fridrichova I, Bujalkova M et al: Novel hMLH1 and $h M S H 2$ germline mutations in the first HNPCC families identified in Slovakia. Hum Mutat 2003; 21: 449-453.

19 Zavodna K, Bujalkova M, Krivulcik T et al: Novel and recurrent germline alterations in the MLH1 and MSH2 genes identified in hereditary nonpolyposis colorectal cancer patients in Slovakia. Neoplasma 2006; 53: 269-276.

20 Alemayehu A, Tomkova $\mathrm{K}$, Zavodna $\mathrm{K}$ et al: The role of clinical criteria, genetic and epigenetic alterations in Lynch-syndrome diagnosis. Neoplasma 2007; 54: 393-403.

21 QIAGEN. Multiplex PCR Handbook, p.21, Available at: http:// www1.qiagen.com/literature/handbooks/PDF/PCRAndReverseTranscription/KitsAndEnzymes/PCR_Multiplex/ 1027945_HB_PCR_MP_0804_lr.pdf (accessed 4 April 2007).

22 Giannini G, Ristori E, Cerignoli F et al: Human MRE11 is inactivated in mismatch repair-deficient cancers. EMBO Rep 2002; 3: 248-254.

23 Boland CR: Evolution of the nomenclature for the hereditary colorectal cancer syndromes. Fam Cancer 2005; 4: 211-218.

24 Oda S, Zhao Y, Maehara Y: Microsatellite instability in gastrointestinal tract cancers. Surg Today 2005; 35: 1005-1015.

25 Pawlik TM, Raut CP, Rodriguez-Bigas MA: Colorectal carcinogenesis: MSI-H versus MSI-L. Dis Markers 2004; 20: 199-206.

26 Halford S, Sasieni P, Rowan A et al: Low-level microsatellite instability occurs in most colorectal cancers and is nonrandomly distributed quantitative trait. Cancer Res 2002; 62: $53-57$.

27 Mongiat-Artus P, Miquel C, Van der Aa A et al: Microsatellite instability and mutation analysis of candidate genes in urothelial cell carcinomas of upper urinary tract. Oncogene 2006; 25: $2113-2118$.

28 Paull TT, Lee J-H: The Mre11/Rad50/Nbs1 complex and its role as a DNA double-strand break sensor for ATM. Cell Cycle 2005; 4: 737-740.

29 Takemura H, Rao AV, Sordet O et al: Defective Mre11-dependent activation of Chk2 by Ataxia Telangiectasia mutated in colorectal carcinoma cells in response to replication-dependent DNA double strand breaks. J Biol Chem 2006; 281: 3081430823. 